Original

\title{
Nefrectomia laparoscópica simple con asistencia de notes transvaginal y uso de instrumentos laparoscópicos estándar
}

\author{
Octavio A. Castillo ${ }^{\mathrm{a}, \mathrm{b}, \mathrm{c}}$, Ivar Vidal-Mora ${ }^{\mathrm{a}}$, Rodrigo Campos $^{\mathrm{a}}$, Alejandro Fonerón ${ }^{\mathrm{a}}$, \\ Miguel Feria-Flores ${ }^{a}$, Reinaldo Gómez ${ }^{\mathrm{a}}$, Francisco Sepúlveda ${ }^{\mathrm{a}}$ \\ ${ }^{a}$ Unidad de Urología Clínica Indisa, ${ }^{b}$ Escuela de Medicina, Facultad de Ciencias de la Salud, Universidad \\ Andrés Bello, ${ }^{c}$ Facultad de Medicina, Universidad de Chile, Santiago, Chile.
}

\begin{abstract}
Resumen
Introducción: La cirugía endoscópica transluminal a través de orificios naturales (NOTES ${ }^{\mathrm{TM}}$ ) es una tecnología emergente, que permite realizar procedimientos quirúrgicos sin el uso de incisiones abdominales. Sin embargo existen limitaciones respecto al equipamiento disponible para simular la cirugía tradicional. Nuestro objetivo es presentar nuestra experiencia con la técnica de nefrectomía transvaginal NOTES con el uso de instrumentos laparoscópicos estándar.

Materiales y Métodos: Presentamos dos pacientes de sexo femenino de 23 y 26 años, ambas con diagnóstico de infecciones urinarias recurrentes y atrofia renal secundaria. Se les realizó una nefrectomía simple con asistencia de NOTES, utilizando un puerto de trabajo transvaginal para la cámara y dos puertos adicionales de 10 (umbilical) y $3 \mathrm{~mm}$ en el abdomen.

Resultados: El procedimiento total duró 120 min en el primer caso y 40 minutos en el segundo, con una pérdida sanguínea promedio de 200 cc. (0-200). No se reportan complicaciones, siendo ambas pacientes dadas de alta 36 horas después de su cirugía.

Conclusión: La nefrectomía laparoscópica con asistencia de NOTES transvaginal es técnicamente posible con el uso de instrumentos laparoscópicos estándar. El desarrollo y acceso a trócares y pinzas especiales para la técnica NOTES ${ }^{\mathrm{TM}}$, permitirá realizar una técnica pura sin el uso de incisiones abdominales.
\end{abstract}

Palabras clave: Cirugía mínimamente invasiva. Nefrectomía. NOTES.

\section{Laparoscopic simple nephrectomy with transvaginal notes assistance and the use of standard laparoscopic instruments}

\section{Abstract}

Introduction: Natural orifice transluminal endoscopic surgery (NOTES) is an emerging technique, which allows to performed surgical procedures avoiding any surgical scars. However there are some problems due to the lack of equipment available for these procedures. The aim of these study is to present our initial experience with the transvaginal nephrectomy NOTES using standard laparoscopic instruments.

Material and Methods: Two female patients 23 and 29 years old, both of them with diagnosis of recurrent urinary tract infection and renal atrophy. A transvaginal simple nephrectomy was performed using a transvaginal Access for the camera port and two abdominal work ports of 10 and $3 \mathrm{~mm}$.

Results: Total operation room time was $120 \mathrm{~min}$ in the first case and $40 \mathrm{~min}$. in the second. with an average blood loss of $200 \mathrm{cc}$. There were no perioperative complications, and both patients was discharged 36 hours after the surgery

Conclusion: Laparoscopic nephrectomy with transvaginal NOTES assistance is technically feasible with the use of standard laparoscopic instruments. Special Access trocars and instruments development for this procedure will allow to perform a pure technique without the use of abdominal incisions.

Keywords: Minimally invasive surgery. Nephrectomy. NOTES.

$\mathrm{L}$ a cirugía endoscópica a través de orificios naturales es una tecnología emergente que combina los principios de la endoscopía y la laparoscopía para realizar cirugía tradicional sin el uso de incisiones abdominales ${ }^{1,2}$. A diferencia de la cirugía abierta y laparoscópica tradicional, el acceso a la cavidad abdominal es primero establecido por el paso de los equipos endoscópicos a través de los orificios naturales. Se crea una incisión transvisceral controlada y se pasa el equipamiento endoscópico en la cavidad peritoneal permitiendo la visualización de los órganos intraabdominales. Gettman et $\mathrm{al}^{3}$. 
reportaron su experiencia en el año 2002 con una nefrectomía transvaginal en modelo porcino, usando instrumentos laparoscópicos estándar. Posteriormente en el año 2008 Branco et $\mathrm{al}^{4}$. describen una nefrectomía transvaginal híbrida con el uso de 2 puertos abdominales en una paciente con riñón no funcionante. El concepto de NOTES ${ }^{\mathrm{TM}}$ avanza a pasos agigantados, pero requiere el uso de instrumental adecuados. Presentamos nuestra experiencia con la técnica de nefrectomía laparoscópica transvaginal con asistencia de $\operatorname{NOTES}^{\mathrm{TM}}$ y el uso de instrumentos laparoscópicos estándar.

\section{MATERIAL Y MÉTODOS}

Dos pacientes de sexo femenino de 23 y 26 años de edad, ambas con diagnóstico de infecciones urinarias recurrentes. El estudio demostró un riñón izquierdo atrófico e hidronefrótico en el primer caso y un riñón atrófico en el segundo. Se planificó nefrectomía laparoscópica con asistencia de NOTES $^{\mathrm{TM}}$ transvaginal. El procedimiento fue explicado plenamente a las pacientes y su familia, los cuales entregaron su consentimiento.

Descripción de la técnica: Bajo anestesia general, la paciente fue ubicada en posición de flanco izquierdo asociada a posición de litotomía dorsal (Fig. 1). Previamente se instaló sonda nasogástrica y sonda Foley, administrándose antibióticos profilácticos.

Se instaló un trócar de $10 \mathrm{~mm}$ en el ombligo, estableciendo pneumoperitoneo hasta $15 \mathrm{mmHg}$. Bajo visión laparoscópica, se instaló una trocar de $10 \mathrm{~mm}$. a través de del fondo de saco vaginal posterior. Este puerto fue utilizado posteriormente para la introducción de la óptica de $30^{\circ}$ (Fig. 2). La cavidad abdominal fue inspeccionada y finalmente un

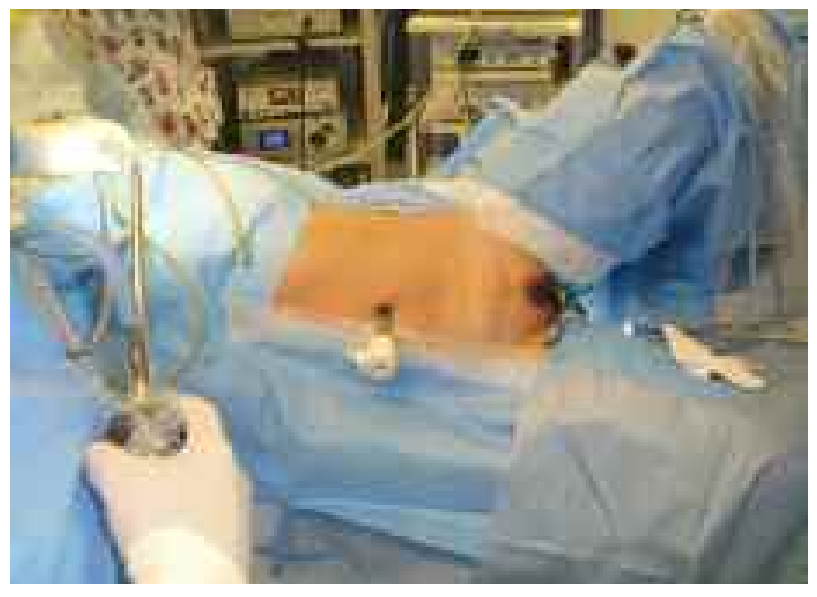

FIGURA 1

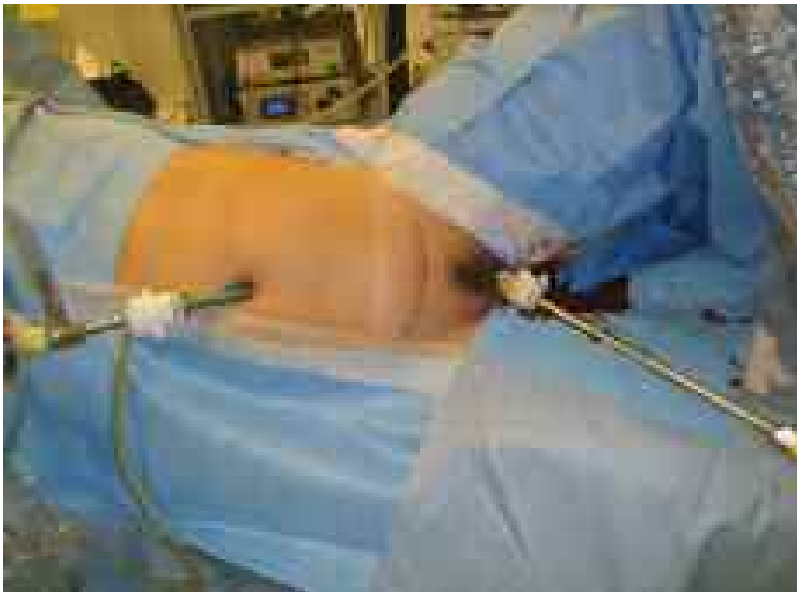

FIGURA 2

puerto de $3 \mathrm{~mm}$. se instaló en el cuadrante superior izquierdo (Fig. 3). La disección fue realizada a través de visión laparoscópica transvaginal, con el uso de instrumentos laparoscópicos estándar. Los pasos de la nefrectomía clásica fueron replicados sin inconvenientes y el espécimen quirúrgico fue capturado en el sistema de recuperación Monarch ${ }^{\circledR}$. Posteriormente la pieza quirúrgica fue extraída por vía transvaginal, utilizando la colpotomía por la que se insertó el trocar de trabajo. La mucosa vaginal fue cerrada con una sutura absorbible $2 / 0$.

\section{RESULTADOS}

El procedimiento completo duró 120 minutos en el primer caso y 40 minutos en el segundo paciente. La pérdida sanguínea promedio fue de 200 cc. (0200). Ambas pacientes evolucionaron sin complicaciones. La alimentación se reinició a la mañana siguiente del procedimiento y fueron dadas de alta a las 36 h (Fig. 4).

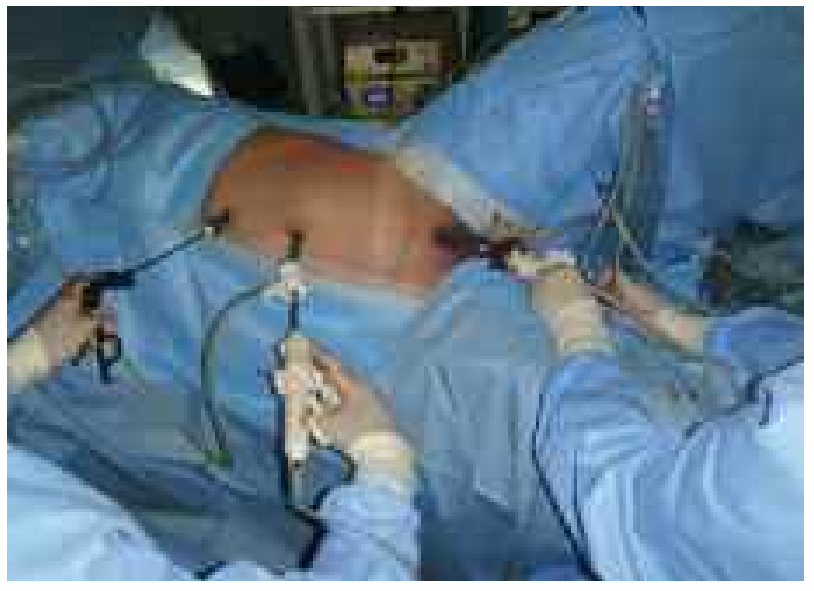

FIGURA 3 


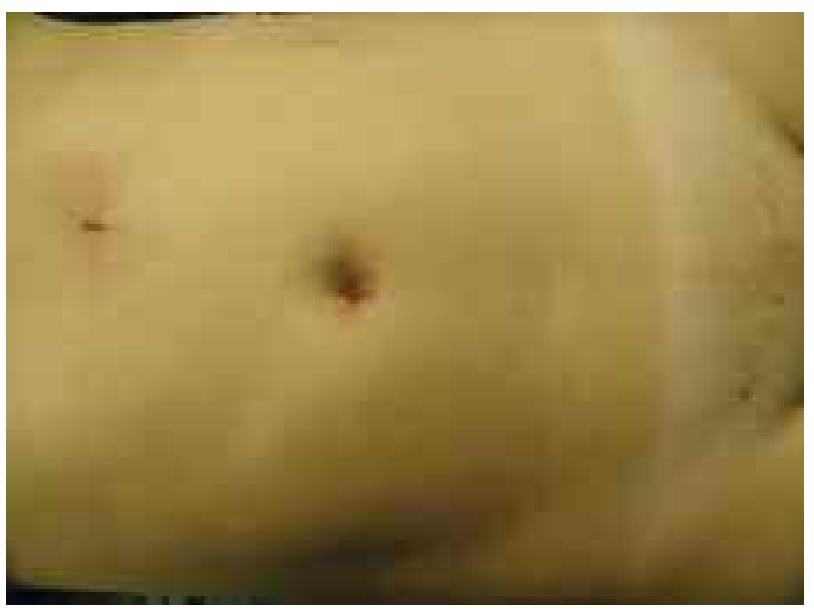

FIGURA 4

El análisis histopatológico demostró pielonefritis crónica y atrofia renal.

\section{DISCUSIÓN}

La primera serie de NOTES ${ }^{\mathrm{TM}}$ fue reportada en el 2004 por Kalloo et $\mathrm{al}^{5}$, describiendo la técnica de biopsia hepática por vía transgátrica. Después de esta publicación otros autores han demostrado la factibilidad de la vía transgástrica para realizar ligadura de la trompas de Falopio $^{6}$, colecistectomia ${ }^{7}$, gastroyeyunoanastomosis ${ }^{8}$, histerectomía parcial con ooforectomía ${ }^{9}$, esplenectomía ${ }^{10}$ y nefrectomía ${ }^{11}$, todas basadas en experiencias en modelos porcinos.

En abril de 2007, Marescaux et al. ${ }^{12}$ reportaron la primera cirugía sin cicatrices, llamada "Operación Anubis". Se realizó una colecistectomía transvaginal con un endoscopio flexible, asociado a un puerto de $2 \mathrm{~mm}$ para la instilación de gas y la medición de la presión intra abdominal.

Las ventajas conocidas de la laparoscopía tradicional sobre la cirugía abierta, han llevado a los cirujanos minimamente invasivos a esperar beneficios adicionales de NOTES ${ }^{\mathrm{TM}}$ sobre la laparoscopía:

1) Ausencia de incisiones abdominales

2) Reducción del dolor post operatorio

3) Posibilidad de una anestesia diferente a la general

4) Ventajas en pacientes obesos o con otros defectos de la pared abdominal

5) Disminución del riesgo post operatorio de hernias incisionales

6) Facilidad de acceso $^{13}$

7) Otros aspectos como recuperación más temprana y menos íleo post-operatorio.
La vagina es considerada una ruta viable para la extracción del riñón después de una nefrectomía laparoscópica. Este acceso permite mejorar los resultados cosméticos y minimizar la morbilidad, cuando se compara a la remoción a través de incisión abdominal ${ }^{14}$.

Nuestro reporte demuestra la factibilidad de la nefrectomía laparoscópica usando NOTES ${ }^{\mathrm{TM}}$ con acceso vaginal, no sólo para la extracción del espécimen quirúrgico, sino que también como puerto de trabajo, que en nuestro caso fue utilizado para la introducción de la óptica

Si bien la utilización de un puerto transumbilical y otro auxiliar de $3 \mathrm{~mm}$ en el cuadrante superior izquierdo no cumple con la definición pura de NOTES ${ }^{\mathrm{TM}}$, hoy en día la utilización de un orificio natural como puerto adicional para la cirugía laparoscópica ya sea para la introducción de un instrumento o de un endoscopio para visualización, se enmarca en lo que se conoce como cirugía NOTES ${ }^{\mathrm{TM}}$ asistida $^{15}$. Estas definiciones son de vital importancia para evaluar objetivamente y entender la utilidad clínica final de estos novedosos accesos.

Es importante diferenciar nuestra experiencia del concepto de LESS (Laparoendoscopic single site surgery). Este acceso involucra la realización de la cirugía a través de una incisión/localización única, ya sea con un puerto único, múltiples puertos o una plataforma única multi-puerto ${ }^{15} \mathrm{Al}$ respecto, Sotelo et $\mathrm{al}^{16}$. presentaron su experiencia con la técnica de LESS en una serie de 28 pacientes, destacando la realización de 20 prostatectomías simples transvesicales, 5 histerectomías y una nefrectomía simple. Los resultados son alentadores.

La realización de este acceso todavía es lejano para nuestras posibilidades, ya que continuamos teniendo dificultades para obtener cualquiera de los puertos de trabajo y los instrumentos articulados disponibles en el mercado. Esto último nos llevó a verificar nuestras posibilidades con los instrumentos disponibles con el fin de continuar nuestra experiencia.

$\mathrm{Al}$ analizar la literatura, sólo se han reportado 7 casos en humanos de nefrectomía a través de un puerto único transumbilical, lo que se enmarca en la definición de LESS y un caso nefrectomía híbrida transvaginal (Tabla 1).

A pesar de que esta cirugía parece ser prometedora, sabemos claramente que muchos aspectos, sobre todo en lo concerniente a la instrumentación 
Tabla 1. Principales experiencias clínicas en Nefrectomía con técnica de NOTES y LESS

\begin{tabular}{lcclll}
\hline Autor & Año de publicación & Técnica & Tipo de Cirugia & n & Observación \\
\hline Gill et al. $^{19}$ & 2008 & LESS & Nefrectomía del donante & 5 & Uso de R-Port \\
Desai et al. ${ }^{20}$ & 2008 & LESS & Nefrectomía & 1 & Uso de R-Port \\
Sotelo et al. ${ }^{16}$ & 2009 & LESS & Nefrectomía simple & 1 & Uso de R-Port \\
Branco et al. ${ }^{4}$ & 2008 & NOTES & Nefrectomía simple & 1 & NOTES híbrido transvaginal \\
Castillo et al. & 2009 & NOTES & Nefrectomía simple & 2 & Asistencia de NOTES transvaginal y uso de \\
& & & & instrumentos laparoscópicos estándar \\
\hline
\end{tabular}

*NOTES ${ }^{\mathrm{TM}}$ : Cirugía endoscópica transluminal a través de orificios naturales; LESS: Laparoendoscopic single site surgery; R-Port: Plataforma multi-puerto

necesitan ser mejorados, antes de una implementación masiva de esta tecnología ${ }^{17}$. Sin embargo como en los primeros días de la cirugía laparoscópica, algo que se pensó como futurista, es hoy en día un concepto estándar en nuestra realidad quirúrgica. En la medida que la tecnología avanza, creemos que abordajes aún con menos morbilidad quirúrgica serán desarrollados a una escala impensada. Finalmente creemos que la plataforma de NOTES ${ }^{\text {TM }}$ es un complemento ideal para la cirugía robótica, como ya han demostrado algunas series iniciales ${ }^{18}$.

\section{CONCLUSIÓN}

La nefrectomía simple transvaginal NOTES ${ }^{\mathrm{TM}}$ con el uso de instrumentos laparoscópicos estándar fue llevada a cabo con éxito y sin complicaciones. Se presenta ante nosotros una nueva frontera en la cirugía renal mínimamente invasiva, donde el cirujano crea un abdomen libre de cicatrices para el paciente, usando ya sea orificios embriológicos como el ombligo $\mathrm{u}$ orificios naturales como la vagina.

\section{REFERENCIAS}

1. Rattner D, Kalloo A; ASGE/SAGES Working Group. ASGE/SAGES Working Group on Natural Orifice Translumenal Endoscopic Surgery. Surg Endosc 2006;20(2):329-333.

2. McGee MF, Rosen MJ, Marks J, Onders RP, Chak A, Faulx A, et al. A primer on natural orifice transluminal endoscopic surgery: Building a new paradigm. Surg Innov 2006;13(2):86-93.

3. Gettman MT, Lotan Y, Napper CA, Cadeddu JA. Transvaginal laparoscopic nephrectomy: Development and feasibility in the porcine model. Urology 2002;59(3):446-450.

4. Branco A, Branco Filho A, Kondo W. Hybrid Transvaginal Nephrectomy. Eur Urol 2008; 53: 1290-1294.

5. Kalloo AN, Singh VK, Jagannath SB. Flexible transgastric peritoneoscopy: a novel approach to diagnostic and therapeutic interventions in the peritoneal cavity. Gastrointest Endosc 2004;60(1):114-117.

6. Jagannath SB, Kantsevoy SV, Vaughn CA. Peroral transgastric endoscopic ligation of fallopian tubes with long-term survival in a porcine model. Gastrointest Endosc 2005;61(3):449-453.

7. Park PO, Bergstro"mM, Ikeda K. Experimental studies of transgastric gallbladder surgery: cholecystectomy and cholecystogastric anastomosis (videos). Gastrointest Endosc 2005;61(4):601-616.
8. Kantsevoy SV, Jagannath SB, Niiyama H. Endoscopic gastrojejunostomy with survival in a porcine model. Gastrointest Endosc 2005;62(2):287-292.

9. Wagh MS, Merrifield BF, Thompson CC. Endoscopic transgastric abdominal exploration and organ resection: initial experience in a porcine model. Clin Gastroenterol Hepatol 2005;3 (9):892-8966.

10. Kantsevoy SV, Hu B, Jagannath SB. Transgastric endoscopic splenectomy: is it possible? Surg Endosc 2006;20(3):522-525.

11. Clayman RV, Box GN, Abraham JB. Rapid communication: transvaginal single-port NOTES nephrectomy: initial laboratory experience. J Endourol 2007;21(6):640-644.

12. Marescaux J, Dallemagne B, Perretta S, Mutter D, Wattiez A, Coumaros D. Operation Anubis: first "no scar" surgery. www.websurg.com/event/Anubis/anubis_presse_en.pdf, 2007.

13. Swain P. A justification for NOTES -natural orifice translumenal endosurgery. Gastrointest Endosc 2007;65(3):514-516.

14. Gill IS, Cherullo EE, Meraney AM. Vaginal extraction of the intact specimen following laparoscopic radical nephrectomy. J Urol 2002;167(1):238-241.

15. Box G, Averch T, Cadeddu J, Cherullo E, Clayman R. Nomenclature of Natural Orifice Translumenal Endoscopic Surgery (NOTES ${ }^{\mathrm{TM}}$ ) and Laparoendoscopic Single-Site Surgery (LESS) Procedures in Urology. J Endourol 2008;22(1):25752581.

16. Sotelo R, Astigueta JC, Carmona O, De Andrade R, SanchezSalas R. Laparo-endoscopia por acceso único: experiencia inicial. Actas Urol Esp. 2009;33(2):172-181.

17. Castillo O, Sánchez-Salas R, Vidal-Mora I. NOTES in Urology: Where we are and where we are going. Arch Esp Urol. 2009; 62(2): 97-101.

18. Box GN, Lee HJ, Santos RJ, Abraham JB, Louie MK, Gamboa AJ, et al. Rapid Communication: Robot assisted NOTES nephrectomy: Initial report. J Endourol 2008;22(3):503-506.

19. Gill IS, Canes D, Aron M, Haber GP, Goldfarb DA, Flechner S, et al. Single port transumbilical E-NOTES donor nephrectomy. J Urol 2008;180(2):637-641.

20. Desai MM, Rao PP, Aron M, Pascal-Haber G, Desai MR, et al. Scarless single port transumbilical nephrectomy and pyeloplasty: first clinical report. BJU Int 2008;101(1):83-88.

Correspondencia autor: Dr. Octavio A. Castillo

F.A.C.S.

Av. Apoquindo 3990, Of. 809, Las Condes.

Santiago de Chile. Código postal:7550112

Fax: (56-2) 2282524

E-mail autor: octaviocastillo@vtr.net

Información artículo: Original - Laparoscopia

Trabajo recibido: mayo 2009

Trabajo aceptado: junio 2009 